\title{
Detecting Sub-GeV Dark Matter with Superconducting Nanowires
}

\author{
Yonit Hochberg, ${ }^{1, *}$ Ilya Charaev, ${ }^{2, \dagger}$ Sae-Woo Nam, ${ }^{3, \$}$ Varun Verma, ${ }^{3, \S}$ Marco Colangelo, ${ }^{2, \|}$ and Karl K. Berggren ${ }^{2, \Uparrow}$ \\ ${ }^{1}$ Racah Institute of Physics, Hebrew University of Jerusalem, Jerusalem 91904, Israel \\ ${ }^{2}$ Department of Electrical Engineering and Computer Science, Massachusetts Institute of Technology, \\ Cambridge, Massachusetts 02139, USA \\ ${ }^{3}$ National Institute of Standards and Technology, Boulder, Colorado 80309, USA
}

(Received 12 April 2019; revised manuscript received 3 July 2019; published 10 October 2019)

\begin{abstract}
We propose the use of superconducting nanowires as both target and sensor for direct detection of sub$\mathrm{GeV}$ dark matter. With excellent sensitivity to small energy deposits on electrons and demonstrated low dark counts, such devices could be used to probe electron recoils from dark matter scattering and absorption processes. We demonstrate the feasibility of this idea using measurements of an existing fabricated tungsten-silicide nanowire prototype with $0.8-\mathrm{eV}$ energy threshold and $4.3 \mathrm{ng}$ with $10000 \mathrm{~s}$ of exposure, which showed no dark counts. The results from this device already place meaningful bounds on dark matter-electron interactions, including the strongest terrestrial bounds on sub-eV dark photon absorption to date. Future expected fabrication on larger scales and with lower thresholds should enable probing of new territory in the direct detection landscape, establishing the complementarity of this approach to other existing proposals.
\end{abstract}

DOI: 10.1103/PhysRevLett.123.151802

Introduction.-Dark matter (DM) is one of the most important unsolved mysteries of the Universe. Focus on the weakly interacting massive particle (WIMP) paradigm has guided experimental searches for decades. Traditional methods searching for such weak-scale DM in the laboratory via nuclear recoils have made tremendous progress in probing DM with mass above the $\mathrm{GeV}$ scale, but typically make poor targets for detection of sub-GeV DM that goes beyond the WIMP paradigm. As the WIMP parameter space continues to be covered without discovery of DM, new ideas to search for lighter DM are of the essence.

Indeed, recent years have seen a surge of such new ideas emerge. These include the use of atomic excitations [1], electron recoils in semiconductors [1-4], two-dimensional targets such as graphene [5] and carbon nanotubes [6], color centers [7], and scintillators [8], which can be sensitive to MeV-scale DM masses. Sub-MeV DM can further be probed by superconductors [9-11], Dirac materials [12], superfluid helium [13-15], and polar crystals [16,17]. The proposed experimental designs for each distinct target material differ from one another, with a variety of sensor technology employed across designs, including the use of CCDs, transition edge sensors (TESs), microwave kinetic

Published by the American Physical Society under the terms of the Creative Commons Attribution 4.0 International license. Further distribution of this work must maintain attribution to the author(s) and the published article's title, journal citation, and DOI. Funded by SCOAP ${ }^{3}$. inductance detectors, and graphene field effect transistors (G-FETs) coupled to a target.

Quantum-information science has been breaking new ground in sensor technology, with superconducting nanowires a now established and burgeoning field [18-20]. Some of these nanowires have sub-eV energy sensitivity, which allows them to be used as single-excitation detectors. The recent emphasis on development of such low-threshold, ultrafast, and low-noise single-photon detectors for photonic quantum-information applications [21,22] promises a radical improvement in the search for DM. The advent of superconducting nanowire detectors, which currently have fewer than ten dark counts per day [23] and have demonstrated sensitivity from the midinfrared [24] to the ultraviolet wavelength band [23], provides an opportunity to search for rare low-energy deposits of DM via scattering or absorption processes.

Here we propose and perform initial experiments using this technology as both the target for DM interactions with electrons and the sensor with which to detect these interactions. Depending on the energy thresholds reached in these devices-nanowires with sub-eV thresholds have already been experimentally realized [24] — sensitivity to low-mass DM can be achieved. Energy deposits of order of a few $\mathrm{eV}$ and above can further allow for directional detection of DM via a stacked geometry, which would serve as a powerful discriminate between signal and background.

In this Letter, we begin by describing the basic detection process in these devices. We then describe an existing prototype nanowire and report on how it can be used to 


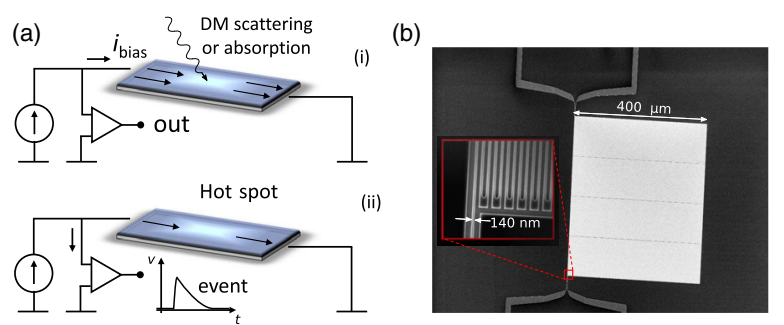

FIG. 1. (a) Schematic depiction of the operating principle of SNSPDs: (i) The detector is biased at a current close to the critical value. (ii) When the energy is absorbed by the nanowire, the electrons depart from equilibrium and diffuse out of the formed hot spot. A resistive region formed across the nanowire then leads to a measurable voltage pulse in the readout. (b) The SEM image of the prototype WSi device after fabrication. The active area is $400 \times 400 \mu \mathrm{m}^{2}$. Nanowires are consistently connected to two contact pads.

extract new bounds on DM interactions with electrons, both in scattering and absorption processes. Our projections for the future reach of superconducting nanowires into the DM parameter space follow. We conclude with a discussion of impact, remaining issues, and possible future work.

Concept.-Superconducting nanowires are a rapidly developing technology with applications ranging from space communications [25,26], to LIDAR [27,28], to quantuminformation science [29]. With sub-eV energy sensitivity [24], $\sim 10^{-4}$ counts/s dark count rates for a device with area $56 \mu \mathrm{m}^{2}$ [23], and spatial discrimination ability [30], superconducting-nanowire single-photon detectors (SNSPDs) provide an excellent candidate for detecting DM. SNSPDs are fabricated by using superconducting films a few nanometers thick on a variety of substrates, with widths between 30 and $200 \mathrm{~nm}$ using electron-beam lithography and reactive ion etching. SNSPDs are typically fabricated into planar meander structures covering tens to hundreds of square micrometers [31]. The device operating principle is straightforward: when cooled below the superconducting transition temperature and biased with a sufficiently high current, the energy deposited by an incident particle can cause the transition of a portion of the nanowire into the normal (resistive, nonsuperconducting) state. This appearance of a resistive region in the current-biased nanowire results in voltage pulses with typical amplitudes of $\sim 1 \mathrm{mV}$ (depending on the amplifier's input impedance) and durations of a few to tens of nanoseconds. A schematic depiction of the device operation is shown in Fig. 1(a). These detectors have demonstrated dark count rates as low as $1 \times 10^{-4}$ counts $/ \mathrm{s}$ [23], making them particularly interesting for sensing rare events.

We therefore propose the use of SNSPDs for direct detection of DM. They can be used as both the target material with which the DM interacts, as well as the sensitive sensor measuring this interaction. Large target mass can be achieved via large arrays combined with multiplexing [32], without disturbing the excellent energy threshold of these devices nor their low-noise character.

A useful rule of thumb regarding the connection between the energy threshold of the device versus the DM mass that it can probe is as follows. In a DM scattering process off a target, the maximal energy deposited is the entire kinetic energy the particle is carrying $\sim m_{\mathrm{DM}} v_{\mathrm{DM}}^{2}$, where $m_{\mathrm{DM}}$ and $v_{\mathrm{DM}}$ are the DM mass and velocity, respectively. Since the DM velocity around us is of order $10^{-3}$ in natural units (where $c=\hbar=1$ ), a given system sensitive to energy deposits of $E_{D}$ or larger can probe DM masses $10^{6}$ larger than $E_{D}$ via the scattering process, $E_{D}^{\text {scat }} \sim 10^{-6} m_{\mathrm{DM}}$. If instead the DM particle is absorbed by the target, it deposits its entire mass energy, meaning that the same target system is sensitive to $E_{D}^{\mathrm{abs}} \sim m_{\mathrm{DM}}$ via absorption processes.

For DM scattering with electrons in the SNSPDs, devices with eV-scale thresholds can thus probe DM mass of $\mathrm{MeV}$ and above. In this mass range, several proposed other targets exist in the literature (see, e.g., Ref. [33] for a recent community report). The reach of the SNSPDs can be comparable to or better than these other targets, depending on exposure size and duration, and is complementary to other approaches. The SNSPDs, however, offer the advantage of possible directionality of the signal: with energy deposits of a few eV and above, the electrons are likely to be ejected from the material and could then hit multiple layers of SNSPD arrays. If it is found that the ejected electron from the superconductor tracks the direction of the incoming DM particle [34], then reproducing the direction of the outgoing electron via the stacked geometry and the SNSPD's spacial discrimination power would inform us about the directionality of the signal. This could also help discriminate signal from background. Similar use of directionality from a stacked configuration has been suggested for use in graphene targets [5].

As the threshold of the device is lowered to sub-eV energies, lower DM masses can be probed, with $\mathcal{O}(\mathrm{meV})$ energy deposits above the superconducting gap corresponding to $\mathcal{O}(\mathrm{keV}) \mathrm{DM}$ masses. Indeed, nanowires that exhibit sensitivity to $5 \mu \mathrm{m}$ wavelength photons, corresponding to an energy threshold of $\sim 250 \mathrm{meV}$, have been demonstrated [24], and it is likely that further technology developments could push the energy sensitivity to $10 \mu \mathrm{m}$ ( $\sim 125$ meV energies) or even beyond. As we will show, the reach of the SNSPDs into the sub-MeV DM mass range is substantial and can provide excellent results even with very small target masses, which can be constructed on relatively short timescales.

Additionally, as we will show, absorption of DM in the sub-eV and above mass range is similarly possible via SNSPDs, providing an important complementary probe to, e.g., existing stellar constraints.

Existing prototype device.-Having presented the basic concept of detection via SNSPDs, we now describe an existing prototype device and how measurements of its 


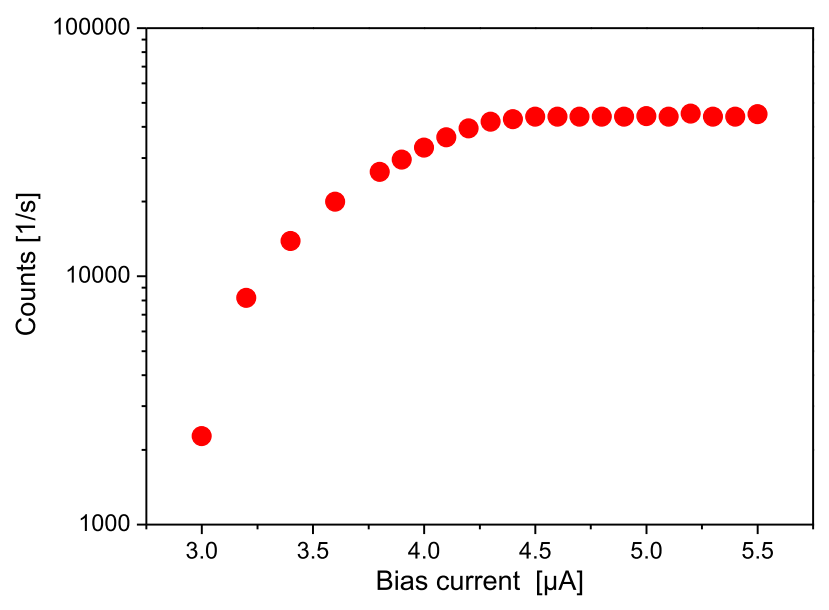

FIG. 2. The photon counts as a function of the absolute bias current, exhibited by the prototype WSi device tested in a fibercoupled package at $300 \mathrm{mK}$.

performance already place bounds on DM scattering and absorption.

Figure 1(b) is a scanning electron micrograph (SEM) of the prototype tungsten silicide (WSi) device after fabrication. The active device area was $400 \times 400 \mu \mathrm{m}^{2}$, and the nanowire was connected to external circuitry via two contact pads. The width of the nanowires was $140 \mathrm{~nm}$ with a pitch of $340 \mathrm{~nm}$. The thickness of the WSi film was $7 \mathrm{~nm}$, and the resulting mass is $4.3 \mathrm{ng}$. Further details of the device design and fabrication are provided in the Supplemental Material [35].

The device was first characterized electrically and optically in an apparatus with a connection to an optical fiber leading outside of the cryostat. The switching current of the device $I_{C}$ was $5.5 \mu \mathrm{A}$ measured at $300 \mathrm{mK}$ by sweeping the current from a $50 \Omega$-impedance source. Figure 2 shows the dependence of the count rate on the absolute bias current for this $400 \times 400 \mu \mathrm{m}^{2}$ large-area SNSPD at $1550 \mathrm{~nm}$ wavelength $(\sim 0.8 \mathrm{eV})$. When the detector was illuminated, the count rate rose at a bias current of $3 \mu \mathrm{A}$. Counts initially grew with the current and the device saturated at a bias current of $4.5 \mu \mathrm{A}$. At this bias current, the count rate with the laser light turned off (background count rate, presumably originating from infrared blackbody illumination guided through fiber) was below 100 counts/s. The maximum background count rate was measured at a point just below the transition to the resistive state, at $10^{3}$ counts/s.

To eliminate the effect of blackbody illumination from the optical fiber, the optical fiber connection was then removed, several layers of shielding were added, and the device was retested. To establish that the detector was still operating correctly, the device was biased to just below its critical current and background events were again observed
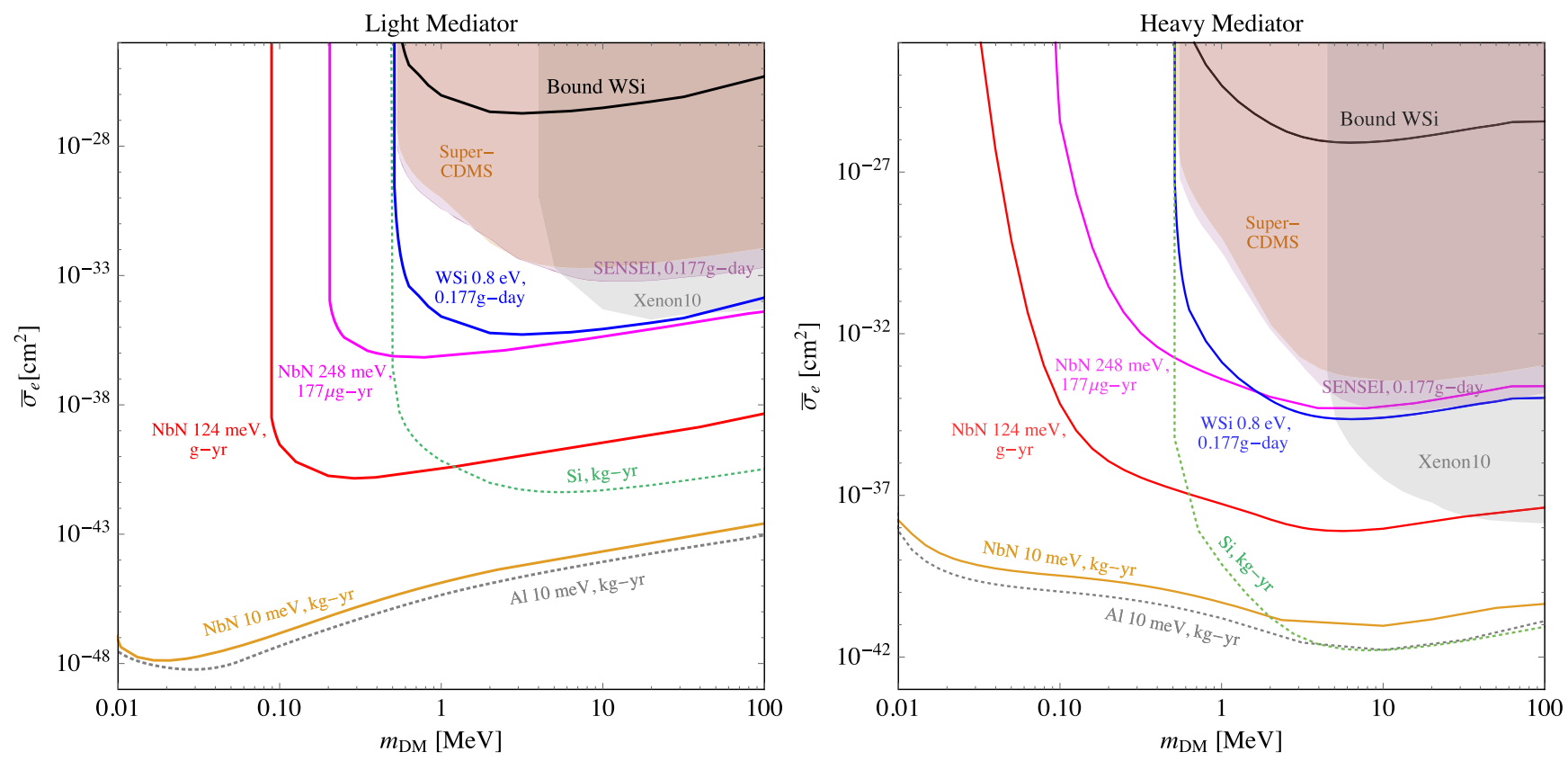

FIG. 3. Expected reach for DM-electron scattering via a light (left) and heavy (right) mediator as a function of DM mass. The solid black curve labeled "Bound WSi" indicates the new bound placed by our prototype device with $4.3 \mathrm{ng}$ exposed for $10000 \mathrm{~s}$. Other solid curves indicate our background-free 95\% C.L. projected reach for either NbN or WSi targets, with various exposures and thresholds. Also shown are the existing constraints from Xenon10 [2] (shaded gray), SuperCDMS [36] (shaded red), and SENSEI [37] (shaded purple), as well as the projected reach for a $\mathrm{kg}$ yr exposure of a silicon target [38] (dotted green) and superconducting bulk aluminum with a $10 \mathrm{meV}$ threshold $[9,10]$ (dotted gray). For clarity, $177 \mu \mathrm{g}$ corresponds to a $10 \times 10 \mathrm{~cm}^{2}$ area of $\mathrm{NbN}$ at $4 \mathrm{~nm}$ thickness and a $50 \%$ fill factor, and a 248 (124) $\mathrm{meV}$ threshold corresponds to a 5(10) $\mu \mathrm{m}$ wavelength. 
(presumably due to low-energy blackbody photons or intrinsic thermal or quantum fluctuations in the device).

Finally, the main experimental run was performed. In this experiment, the bias current was set to $4.5 \mu \mathrm{A}$ for $10^{4} \mathrm{~s}$. No counts were observed over this period, suggesting a count rate below 100 counts per megasecond. These measurements will be used below to place bounds on DM interactions.

Reach.-Our results for the reach of superconducting nanowires into the parameter space of DM-electron scattering are shown in Fig. 3. We follow the analyses of Refs. $[9,10]$ for rate computation in superconducting targets, with the appropriate modifications to Fermi energies $E_{F}$ and the density $\rho$ of target materials that are typically used for the superconductors of SNSPDs. Details of the scattering rate computation can be found in the Supplemental Material [35]. Our results for niobium nitride $(\mathrm{NbN})$ and WSi targets use $\rho_{\mathrm{NbN}}=8.4$ and $\rho_{\mathrm{WSi}}=9.3 \mathrm{~g} / \mathrm{cm}^{3}$, respectively, and in both cases we use $E_{F}=7 \mathrm{eV}$. (We note that, while the Fermi surface of WSi is not a perfect sphere, the calculations performed here are intended to provide a proxy to guide future experiments; we have thus assumed a spherical Fermi surface in the case of WSi.)

The left panel of Fig. 3 shows the reach for scattering via a light mediator, with the commonly used reference momentum $q_{\text {ref }}=\alpha m_{e}$ defining the reference cross section $\bar{\sigma}_{e}$, while the right panel shows the reach when scattering via a heavy mediator (see Supplemental Material [35] for all definitions). The solid colored curves show the background-free 95\% confidence level (C.L.) projected reach, corresponding to three signal events, for SNSPDs with various amounts of exposures and thresholds, assuming a dynamic range of 3 orders of magnitude. We also show the projected reach for a $\mathrm{kg}$ yr exposure of a silicon target with single-electron sensitivity [38] and of an aluminum target with a $10 \mathrm{meV}$ threshold TES $[9,10]$, along with constraints from the Xenon10 [2], SENSEI [37], and SuperCDMS [36] experiments.

The 95\% C.L. bound on DM-electron scattering placed by the $4.3 \mathrm{ng}$ prototype $\mathrm{WSi}$ device with the $0.8 \mathrm{eV}$ energy threshold presented in this Letter, which showed no dark counts in $10000 \mathrm{~s}$ of exposure, is shown by the black solid curve. While the bound from this prototype nanowire on DM-electron scattering is not yet competitive with those from the other experiments, it is impressively placed using a tiny mass-time exposure on a surface run. For comparison, the projected reach of a WSi target with an exposure similar to that of the SENSEI data, $0.177 \mathrm{~g}$ day, is also shown, demonstrating the strength of our proposal. As is evident, larger exposures combined with low thresholds will enable superconducting nanowires to quickly probe uncharted parameter space of DM scattering.

In addition to probing DM-electron scattering, SNSPDs can simultaneously probe absorption of relic particles that interact with electrons. As an example, we consider a relic

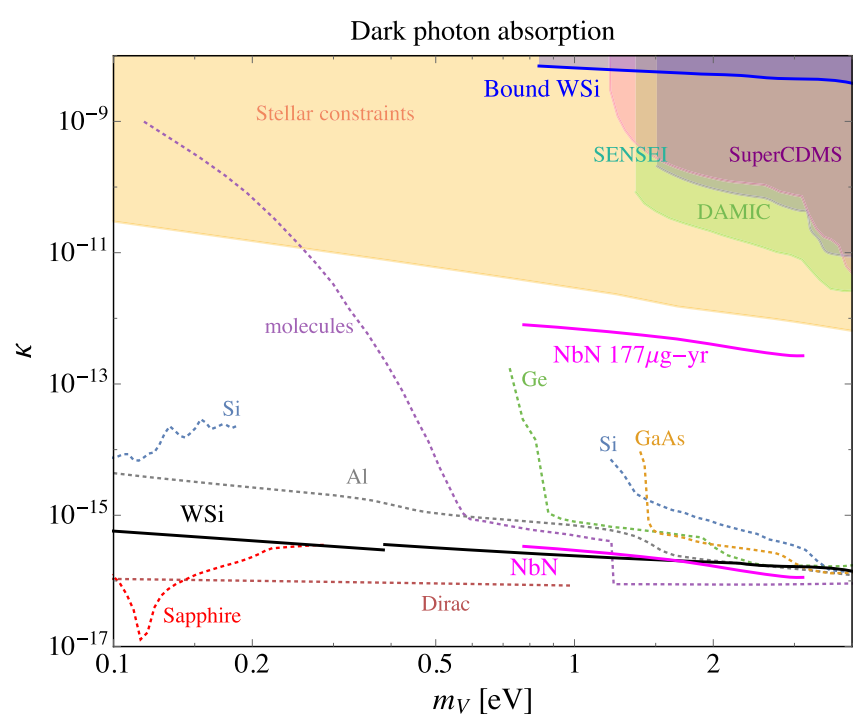

FIG. 4. Expected reach for absorption of relic dark photons. The solid blue curve labeled Bound WSi indicates the new bound placed by our prototype device in this Letter, which showed no dark counts in $4.3 \mathrm{ng}$ over $10^{4} \mathrm{~s}$. The projected reach for a $\mathrm{NbN}$ target SNSPD with $177 \mu \mathrm{g}$ yr (corresponding to a $10 \times 10 \mathrm{~cm}^{2}$ area with $4 \mathrm{~nm}$ thickness and a $50 \%$ fill factor) and $\mathrm{kg} \mathrm{yr}$ exposures is shown (solid magenta curves); $\mathrm{NbN}$ reach into lower masses than depicted should be possible and can be estimated from lower energy data should it become available. Also shown is our projected reach for a $\mathrm{kg}$ yr exposure of a WSi SNSPD (solid black). The reach for a $\mathrm{kg}$ yr exposure of aluminum superconductors [11], semiconductors such as germanium and silicon [44], Dirac materials [12], molecules [45], and polar crystals such as GaAs and sapphire [17] are given as well (dotted curves). Constraints from stellar emission $[42,43]$ are indicated (shaded orange), along with terrestrial constraints from SuperCDMS [36] (shaded purple), DAMIC [41] (shaded green), and SENSEI [37] (shaded turquoise). Unless otherwise stated, projected reach refers to background-free $\mathrm{kg}$ yr exposure.

dark photon that is kinetically mixed with the ordinary photon. Effectively, such a dark photon interacts with electrons in a similar manner to the photon, but with the interaction suppressed by the size of the kinetic mixing $\kappa$ (see Supplemental Material [35] for further details).

Our results for relic dark photon absorption in SNSPDs are shown in Fig. 4. We use low-energy photon absorption data for $\mathrm{NbN}[24]$ and WSi $[39,40]$, and translate it to the expected reach on the size of kinetic mixing $\kappa$ between the photon and dark photon field strengths as a function of the dark photon mass $m_{V}$. Details of the absorption rate computation can be found in the Supplemental Material [35]. We show the resulting background-free 95\% C.L. expected reach, corresponding to three signal events, for a $\mathrm{kg}$ yr exposure of $\mathrm{NbN}$ and WSi target SNSPDs. For NbN, we additionally present the reach of a $177 \mu \mathrm{g}$ yr exposure; we further note that reach into lower masses than depicted is possible and can be estimated should lower energy data become available. Also shown are constraints from 
SuperCDMS [36], DAMIC [41] and SENSEI [37], along with stellar emission constraints [42,43]. The projected reach for $\mathrm{kg} \mathrm{yr}$ exposures of germanium and silicon [44], superconducting aluminum [11], Dirac materials [12], and polar crystals such as GaAs and sapphire [17], as well as molecular targets [45] are likewise indicated. (For the reach of multilayer optical haloscopes, see Ref. [46].)

The $95 \%$ C.L. bound on relic dark photons placed by the data of the $4.3 \mathrm{ng}$ prototype WSi device in $10^{4} \mathrm{~s}$ with a $0.8 \mathrm{eV}$ threshold presented in this Letter is shown by the solid blue curve. Remarkably, despite the small device size and short exposure time of our experiment, it places the strongest terrestrial constraint to date on dark photons with sub-eV masses.

Summary.-We have proposed the use of superconducting nanowires as sensitive targets and detectors for light dark matter and demonstrated the power of this approach. We have found that absorption of bosonic DM with masses above the superconducting gap of $\mathcal{O}(\mathrm{meV})$ and scattering of DM in the keV-GeV mass range are both promising and complementary to other existing proposals in these mass ranges. An existing prototype nanowire already places meaningful bounds on the parameter space, including the strongest terrestrial constraints to date on dark photon absorption in the sub-eV to few-eV mass range.

The results presented here suggest that further work, both theoretical and experimental, is warranted to determine the viability of using SNSPDs for this goal. While SNSPDs are promising candidates for DM detection due to their sub-eV threshold and potential for low dark count rates, they have small masses and do not provide calorimetric information. The former issue might be addressed by scaling the device to larger dimensions by using optical lithography instead of electron lithography, while the latter issue could be partially mediated by using a multipixel configuration [30] or by using photon-number-sensitive SNSPDs [47]. In the Supplemental Material [35], we further elaborate on several issues raised by our results, in particular (i) what are the ideal device characteristics that should be targeted, and (ii) what are the prospects for scaling the detectors to masses large enough to substantially extend the reach of current searches.

The complementarity of different targets, sensors, and experimental detection approaches is important for driving the DM detection field forward. In addition, the development of large arrays of ultralow-noise, ultralow-threshold SNSPDs advocated here could further service other approaches for DM detection, casting a wide net for meaningful improvement to DM detection schemes.

With low thresholds and low dark count rates, superconducting nanowires have the potential to impact the direct detection landscape on relatively short timescales. We hope this Letter serves as a stimulant for broad cooperation between the quantum-information and fundamental physics communities, such that meaningful progress can rapidly be made towards understanding the basic constituents of nature.

We thank Tom Dvir, Nadav Katz, and Hadar Steinberg for useful discussions that led to this work, and Eric David Kramer and Eric Kuflik for many helpful discussions. We thank Phil Mauskopf for providing the cryogenic amplifiers used in this experiment. K. K. B. thanks Asimina Arvanitaki and Ken van Tilburg for helpful discussions. The work of Y. H. is supported by the Israel Science Foundation (Grant No. 1112/17), by the Binational Science Foundation (Grant No. 2016155), by the I-CORE Program of the Planning Budgeting Committee (Grant No. 1937/ 12), by the German Israel Foundation (Grant No. I-2487303.7/2017), and by the Azrieli Foundation. The work of K. K. B. was supported in part by the DOE under the QuantiSED program, Award No. DE-SC0019129.

*yonit.hochberg@mail.huji.ac.il

tcharaev@mit.edu

\#nams@boulder.nist.gov

§varun.verma@boulder.nist.gov

"colang@mit.edu

"berggren@mit.edu

[1] R. Essig, J. Mardon, and T. Volansky, Phys. Rev. D 85, 076007 (2012).

[2] R. Essig, A. Manalaysay, J. Mardon, P. Sorensen, and T. Volansky, Phys. Rev. Lett. 109, 021301 (2012).

[3] P. W. Graham, D. E. Kaplan, S. Rajendran, and M. T. Walters, Phys. Dark Universe 1, 32 (2012).

[4] N. Kurinsky, T. C. Yu, Y. Hochberg, and B. Cabrera, Phys. Rev. D 99, 123005 (2019).

[5] Y. Hochberg, Y. Kahn, M. Lisanti, C. G. Tully, and K. M. Zurek, Phys. Lett. B 772, 239 (2017).

[6] G. Cavoto, F. Luchetta, and A. D. Polosa, Phys. Lett. B 776, 338 (2018).

[7] R. Budnik, O. Chesnovsky, O. Slone, and T. Volansky, Phys. Lett. B 782, 242 (2018).

[8] S. Derenzo, R. Essig, A. Massari, A. Soto, and T.-T. Yu, Phys. Rev. D 96, 016026 (2017).

[9] Y. Hochberg, Y. Zhao, and K. M. Zurek, Phys. Rev. Lett. 116, 011301 (2016).

[10] Y. Hochberg, M. Pyle, Y. Zhao, and K. M. Zurek, J. High Energy Phys. 08 (2016) 057.

[11] Y. Hochberg, T. Lin, and K. M. Zurek, Phys. Rev. D 94, 015019 (2016).

[12] Y. Hochberg, Y. Kahn, M. Lisanti, K. M. Zurek, A. G. Grushin, R. Ilan, S. M. Griffin, Z.-F. Liu, S. F. Weber, and J. B. Neaton, Phys. Rev. D 97, 015004 (2018).

[13] K. Schutz and K. M. Zurek, Phys. Rev. Lett. 117, 121302 (2016).

[14] S. Knapen, T. Lin, and K. M. Zurek, Phys. Rev. D 95, 056019 (2017).

[15] F. Acanfora, A. Esposito, and A. D. Polosa, Eur. Phys. J. C 79, 549 (2019).

[16] S. Knapen, T. Lin, M. Pyle, and K. M. Zurek, Phys. Lett. B 785, 386 (2018). 
[17] S. Griffin, S. Knapen, T. Lin, and K. M. Zurek, Phys. Rev. D 98, 115034 (2018).

[18] C.Natarajan, M.Tanner, and R. Hadfield, Supercond. Sci. Technol. 25, 063001 (2012).

[19] R. Hadfield, Nat. Photonics 3, 696 (2009).

[20] F. Najafi et al., Quantum Sci. Technol. 11, 3 (2007).

[21] H. Takesue, S. W. Nam, Q. Zhang, R. Hadfield, T. Honjo, K. Tamaki, and Y. Yamamoto, Nat. Photonics 1, 343 (2007).

[22] B. Calkins et al., Opt. Express 21, 22657 (2013).

[23] E. Wollman et al., Opt. Express 25, 26792 (2017).

[24] F. Marsili, F. Bellei, F. Najafi, A. E. Dane, E. A. Dauler, R. J. Molnar, and K. K. Berggren, Nano Lett. 12, 4799 (2012).

[25] M. E. Grein, O. Shatrovoy, D. V. Murphy, B. S. Robinson, and D. Boroson, Conference on Lasers and Electro-Optics (CLEO 2014), SM4J.4 (2014).

[26] Y. P. Korneeva, D. Y. Vodolazov, A. V. Semenov, I. N. Florya, N. Simonov, E. Baeva, A. A. Korneev, G. N. Goltsman, and T. M. Klapwijk, Conference on Lasers and Electro-Optics (CLEO 2014), SM4J.5 (2014).

[27] A. McCarthy, N. J. Krichel, N. R. Gemmell, X. Ren, M. G. Tanner, S. N. Dorenbos, V. Zwiller, R. H. Hadfield, and G. S. Buller, Opt. Express 21, 8904 (2013).

[28] L. Chen, D. Schwarzer, V. B. Verma, M. J. Stevens, F. Marsili, R. P. Mirin, S. W. Nam, and A. M. Wodtke, Acc. Chem. Res. 50, 1400 (2017).

[29] C. M. Natarajan, M. M. Härtig, R. E. Warburton, G. S. Buller, R. H. Hadfield, B. Baek, S. W. Nam, S. Miki, M. Fujiwara, M. Sasaki, and Z. Wang, in Quantum Communication and Quantum Networking, edited by A. Sergienko, S. Pascazio, and P. Villoresi (Springer, Berlin, 2010) pp. 225-232.

[30] Q. Zhao, D. Zhu, N. Calandri, A. E. Dane, A. N. McCaughan, F. Bellei, H.-Z. Wang, D. F. Santavicca, and K. K. Berggren, Nat. Photonics 11, 247 (2017).

[31] C. Lv, H. Zhou, H. Li, L. X. You, X. Y. Liu, Y. Wang, W. J. Zhang, S. J. Chen, Z. Wang, and X. M. Xie, Supercond. Sci. Technol. 30, 115018 (2017).
[32] E. A. Dauler, A. J. Kerman, B. S. Robinson, J. K. Yang, B. Voronov, G. Goltsman, S. A. Hamilton, and K. K. Berggren, J. Mod. Opt. 56, 364 (2009).

[33] M. Battaglieri et al., arXiv:1707.04591.

[34] Y. Hochberg, E. D. Kramer, N. Kurinsky, and K. M. Zurek (to be published).

[35] See Supplemental Material at http://link.aps.org/ supplemental/10.1103/PhysRevLett.123.151802 for details of the prototype device, scattering and absorption rates, and detector considerations.

[36] R. Agnese et al. (SuperCDMS Collaboration), Phys. Rev. Lett. 121, 051301 (2018).

[37] O. Abramoff et al. (SENSEI Collaboration), Phys. Rev. Lett. 122, 161801 (2019).

[38] R. Essig, M. Fernandez-Serra, J. Mardon, A. Soto, T. Volansky, and T.-T. Yu, J. High Energy Phys. 05 (2016) 046.

[39] F. Marsili et al., Nano Lett. 7, 210 (2013).

[40] I. Yamada, J. Nishii, and M. Saito, Appl. Opt. 47, 4735 (2008).

[41] A. Aguilar-Arevalo, D. Amidei, X. Bertou, M. Butner, G. Cancelo, A. Castañeda Vázquez1, B. A. Cervantes Vergara, A. E. Chavarria, C. R. Chavez, J. R. T. de Mello Neto et al. (DAMIC Collaboration), Phys. Rev. Lett. 118, 141803 (2017).

[42] H. An, M. Pospelov, and J. Pradler, Phys. Rev. Lett. 111, 041302 (2013).

[43] H. An, M. Pospelov, J. Pradler, and A. Ritz, Phys. Lett. B 747, 331 (2015).

[44] Y. Hochberg, T. Lin, and K. M. Zurek, Phys. Rev. D 95, 023013 (2017).

[45] A. Arvanitaki, S. Dimopoulos, and K. Van Tilburg, Phys. Rev. X 8, 041001 (2018).

[46] M. Baryakhtar, J. Huang, and R. Lasenby, Phys. Rev. D 98 , 035006 (2018).

[47] C. Cahall, K. L. Nicolich, N. T. Islam, G. P. Lafyatis, A. J. Miller, D. J. Gauthier, and J. Kim, Optica 4, 1534 (2017). 